Monográfico / Monographic

\title{
Cuidados comunitarios y gobierno común de la dependencia: las viviendas colaborativas de personas mayores
}

\section{Community care and common governance of dependency: the collaborative housings of the elderly}

\author{
Alba Artiaga Leiras* \\ Universidad Complutense de Madrid, España \\ alba.artiaga.leiras@ucm.es \\ Recibido / Received: 16/01/2020 \\ Aceptado / Accepted: 10/11/2020

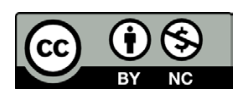

\section{RESUMEN}

Este artículo analiza el papel de la comunidad $y$, en concreto, de las viviendas colaborativas de personas mayores en la reconfiguración de los marcos de sentido en torno a los cuidados, la dependencia y los modos de habitar la vejez. Se parte del análisis del actual modelo de organización de los cuidados y atención a la dependencia que surge con la puesta en marcha de la Ley 39/2006 de Promoción de la Autonomía Personal y Atención a personas en situación de dependencia (LAAD). Será este contexto el que nos permita centrarnos en el estudio de caso del proyecto de vivienda colaborativa Trabensol (Trabajadores en Solidaridad), profundizando así en las tensiones, ambivalencias y posibilidades que ofrece esta fórmula de cara a incorporar una dimensión colectiva a los cuidados y garantizar la soberanía política sobre las vidas de las personas mayores.

Palabras clave: cuidados, comunidad, dependencia, mayores, común.

\section{ABSTRACT}

This paper analyses the role of the community and, more specifically, of collaborative housing for the elderly towards the reshaping process of the meaning frameworks concerning care, dependency and the ways of living old age. The starting point is an analysis of the current organisation model of care of dependent people as of the endorsement of the Law 39/2006 of Promotion of Personal Autonomy and Care of dependent people (LAAD, in its Spanish acronym). This context will allow us to focus on the case study of the collaborative housing project of Trabensol (Workers in Solidarity), thus delving into the tensions, ambivalences and possibilities which stem from this idea with regard to incorporating a collective dimension to care and guaranteeing a political sovereignty of the lives of the elderly.

Keywords: care, community, dependency, the elderly, common. 
Cuidados comunitarios y gobierno común de la dependencia: las viviendas colaborativas de personas mayores

\section{INTRODUCCIÓN}

El progresivo y creciente envejecimiento de la población con el consiguiente aumento de las necesidades de apoyos (Durán, 2018; Carrasco, Borderías y Torns, 2011), la presencia de un modelo de bienestar deficitario, la transformación de las familias y de los roles de género en relación con el cuidar y la desarticulación de los sentidos de pertenencia a lo colectivo, son algunas de las cuestiones que sacan a la luz las dificultades para dar cobertura a las actuales necesidades de cuidados en el contexto español. Dificultades que se hacen aún más evidentes si hablamos de etapas de la vida que requieren de apoyos específicos como puede ser la vejez o de realidades vinculadas con la diversidad funcional.

Con el propósito de atender estas situaciones, en las últimas décadas se impulsan intervenciones políticas de cuidados. Un avance en la politización de los cuidados y la dependencia para el caso español es la Ley 39/2006, de 14 de diciembre, de Promoción de la Autonomía Personal y Atención a personas en situación de dependencia (LAAD)'. Esta ley se considera un hito en la protección social, no obstante, se considera relevante analizar qué maneras de entender y de habitar la dependencia se proponen con la LAAD y hasta qué punto se reconfigura el género del cuidar ya que las políticas públicas de cuidados no tienen por qué ser emancipadoras en si mismas (López y Platero, 2019; Artiaga, 2015; Serrano, Artiaga y Dávila de León, 2013; Bustelo y Lombardo, 2007).

Asimismo, la comunidad juega un papel relevante en la elaboración de fórmulas de cuidados en un contexto de aumento de su demanda y déficits en su provisión (Vega, Martínez y Paredes, 2018). Sin embargo, ha sido un actor tradicionalmente olvidado en los estudios sobre el bienestar y los cuidados, que atienden principalmente a la triada estadomercado-hogares (Daly y Lewis, 2011; Esping-Andersen, 1999). Por ello, se precisa un análisis en profundidad sobre su papel en la configuración de nuevos imaginarios y prácticas que interpelan al actual modelo de cuidados y bienestar. Algunas de las iniciativas que surgen y a las que se presta atención en este artículo son las viviendas colaborativas de personas mayores. En concreto ${ }^{2}$, se profundizará en el estudio de caso del centro de convivencia Trabensol (Trabajadores en solidaridad), ubicado desde el año 2013 en el municipio de Torremocha del Jarama, en la Comunidad de Madrid.

Trabensol es una cooperativa de vivienda impulsada por personas mayores que han tenido una trayectoria de participación conjunta en proyectos sociales y que en la actualidad ensayan maneras de vivir la vejez y de resolver los cuidados de manera colectiva. Tras varios años de definición y elaboración del proyecto construyen un edificio sostenible y preparado para armonizar espacios comunes y espacios de vida independiente, organizándose de manera democrática y promoviendo un clima de cooperación en la resolución de las necesidades cotidianas del bienestar. Además, es un proyecto pionero en dotarse de un Sistema de Atención a la Dependencia complementario al sistema público.

El objetivo de este artículo es analizar qué fórmulas e imaginarios plantea esta iniciativa comunitaria en torno a lo que significa hacerse mayor, habitar la vejez 0 asumir los cuidados como un problema común (Garcés, 2013; Vega et al., 2018). El análisis de las prácticas y los

1 Con aprobación de la Ley 39/2006, de 14 de diciembre, de Promoción de la Autonomía Personal y Atención a personas en situación de dependencia (LAAD) se desarrolla el SAAD (Sistema para la Autonomía y Atención a la Dependencia), que establece un catálogo de servicios y prestaciones económicas para personas mayores y para personas con diversidad funcional que se consideren sujetos de derecho. Los servicios se prestan a través de la oferta pública de la Red de Servicios Sociales o a través de entidades o centros acreditados para la atención a la dependencia y son los siguientes: Servicio de Teleasistencia, Servicio de Ayuda a Domicilio (SAD), Servicio de Centro de Día y Noche, Servicio de Atención Residencial o servicios de prevención de las situaciones de dependencia y promoción de la autonomía personal. Las prestaciones son: prestaciones económicas vinculadas al gasto del servicio en una entidad o centro acreditado, prestaciones económicas para ser atendidos/as por cuidadores/as no profesionales en el entorno familiar o prestaciones económicas de asistencia personal (www.imserso.es).

2 Se utilizarán indistintamente los conceptos de vivienda o alojamiento colaborativo, centro social de convivencia o cooperativa de vivienda de personas mayores. 
marcos de sentido de este proyecto no se puede separar del actual modelo de bienestar en el que se ubica y del auge del paradigma del envejecimiento activo en la regulación de lo social. Por ello, partiendo del contexto de institucionalización de los cuidados y la dependencia para el caso español, se analiza el papel de esta iniciativa en la configuración de escenarios que permitan avanzar hacia la consecución de una vejez digna y la desfeminización y desprivatización de los cuidados.

El artículo se divide en los siguientes apartados: En primer lugar, se analiza la institucionalización de los cuidados y de la dependencia que se promueve con la LAAD para entender el contexto en el que surge esta iniciativa comunitaria. En segundo lugar, se estudia el papel que juega Trabensol en la producción de bienestar y cuidados ampliando los marcos de sentido entorno a lo político, lo económico y lo familiar. En tercer lugar, observamos cómo se redefinen los sentidos en torno a hacerse mayor y habitar la vejez en esta vivienda colaborativa de personas mayores. En cuarto lugar, se profundiza en la dimensión social y la organización colectiva de los cuidados en Trabensol, partiendo del estudio de los mecanismos formales e informales de solidaridad que se ponen en marcha para atender las situaciones de dependencia. Por último, se expondrán brevemente las conclusiones.

\section{DISEÑO METODOLÓGICO}

Este artículo presenta parte de los resultados del proyecto "Cuidados en el ámbito comunitario. Experiencias, prácticas y vínculos para el sostenimiento de la vida en España y América Latina", financiado por el Ministerio de Economía y Competitividad (CSO-201677960-R), cuyo objetivo es estudiar fórmulas de cuidados que se organizan en el ámbito comunitario. Se considera que todavía existe un conocimiento escaso sobre la comunidad como espacio en el que se desarrollan prácticas de cuidados y que es preciso un acercamiento analítico que dé cuenta de su composición, emergencia y despliegue. Esta investigación es eminentemente cualitativa y se extiende entre España, Ecuador y Argentina, con el interés de analizar contextos con fórmulas y valores divergentes en relación con el mercado de trabajo, las políticas públicas, la acción ciudadana o la provisión de cuidados en el ámbito familiar. Para este artículo, se analiza una experiencia comunitaria concreta para el caso español que son las viviendas colaborativas de personas mayores y, específicamente, el caso del centro de convivencia Trabensol. En el marco del actual proyecto se realizan 6 entrevistas (una grupal y cinco individuales) a finales de 2019 (E_1, E_2, E_3, E_4A, E_4B, E_5, E_6), tras seis años de convivencia y una vez puesto en marcha el Sistema de Atención a la Dependencia de Trabensol.

Asimismo, esta investigación sobre el caso de la vivienda colaborativa de personas mayores Trabensol, como ejemplo emblemático de cuidados en la comunidad, da continuidad a una indagación que se inicia con la Tesis doctoral Producción política de los cuidados y de la dependencia: políticas públicas y experiencias de organización social de los cuidados (Artiaga, 2015). Se realiza en el marco de dos proyectos de investigación sobre el gobierno de la dependencia y los cuidados con la LAAD: FEM2010-18827, financiado por el Ministerio de Ciencia e Innovación y 4164391-8/10, con apoyo económico del Instituto de la Mujer. En ellos se lleva a cabo una metodología cualitativa a partir del análisis discursivo de entrevistas a 22 actores clave que participan en el diseño de la LAAD y del análisis de marcos interpretativos de la Ley 39/2006 de Autonomía Personal y Atención a Personas en Situación de Dependencia (LAAD) y de las regulaciones que la acompañan (Acuerdos, Reales Decretos, Dictámenes, etc.). Este análisis nos permite ubicar el contexto en el que se pone en marcha la iniciativa de Trabensol y los marcos discursivos con los que se da sentido a los cuidados y a la dependencia con el desarrollo de la LAAD. Además, se realizan 10 estudios de caso de experiencias de implementación de esta política pública en los que se entrevista a 43 sujetos con diversos perfiles sociodemográficos (cuidadoras/es familiares, profesionales, personas con diversidad funcional, mayores, otros familiares). Uno de 
los estudios de caso seleccionados es el centro de convivencia para personas mayores Trabensol, que se considera un caso atípico por ser una experiencia de autoorganización de los cuidados desde la comunidad. Entre 2011 y 2013 se realizan 3 entrevistas en Trabensol (E_7, E_8, E_9), antes de la construcción y al poco tiempo de entrar a convivir en el centro social de mayores.

En este artículo se considera el estudio de caso como la técnica más adecuada para el análisis en profundidad de la experiencia de Trabensol. El estudio de caso como técnica permite identificar las relaciones de conflicto, las vivencias cruzadas y los procesos de interacción entre los diferentes participantes de una misma situación social. Facilita el acercamiento al carácter multivocal de esta iniciativa, recuperando las diferentes experiencias a fin de no atender únicamente a identidades unitarias (personas mayores) sino a la interacción, las relaciones de conflicto y de complementariedad entre los diversos modos de vivir, así como de entender la vejez y los cuidados en el seno de este proyecto comunitario (Yin, 1984).

Se incluyen distintos tipos de perfiles a partir de los cuáles se pueden observar las diferentes disposiciones, representaciones y vivencias atravesadas por el género, el perfil profesional, el tiempo de participación en el proyecto, el tipo de convivencia (individual o en pareja) y el tipo de participación en Trabensol (véase Tabla 1). En relación con el tipo de participación en el proyecto se seleccionan algunos perfiles vinculados con la comisión de dependencia por la relación con el objeto de estudio. Además, los distintos momentos en los que se realizan las entrevistas (2011, 2013 y 2019) nos dan claves sobre las tensiones que se dan entre los objetivos de partida y la realidad de la experiencia en el día a día, así como las ambivalencias entre el decir y el hacer, es decir, entre los discursos sobre el proyecto y las prácticas concretas (Martín Criado, 1998). Las personas entrevistadas tienen en común la franja de edad, entre 65 y 75 años aproximadamente. Además, son personas con trayectorias activistas e involucradas en proyectos cooperativos y sociales o cercanas a ellos, como el Colegio cooperativo Siglo XXI, la cooperativa de vivienda TRABENCO (Trabajadores en cooperativa) o comunidades cristianas de base. La mayoría de las personas entrevistadas son de Madrid, muchas de ellas de Moratalaz y Vallecas, excepto una persona entrevistada que es de Barcelona. 
Tabla 1. Perfiles de personas entrevistadas

\begin{tabular}{|c|c|c|c|c|c|}
\hline $\begin{array}{l}\text { Código/ } \\
\text { fecha }\end{array}$ & Género & $\begin{array}{c}\text { Perfil } \\
\text { profesional }\end{array}$ & $\begin{array}{l}\text { Tiempo de } \\
\text { participación }\end{array}$ & $\begin{array}{c}\text { Tipo de } \\
\text { participación } \\
\text { (Comisión) }\end{array}$ & $\begin{array}{c}\text { Tipo de } \\
\text { convivencia }\end{array}$ \\
\hline E1_2019 & Mujer & Psicóloga & $\begin{array}{c}\text { Desde } 2014 \\
\text { (discontinuo) }\end{array}$ & Dependencia & Individual \\
\hline E2_2019 & Mujer & Matrona & Desde el inicio & Dependencia & Individual \\
\hline E3_2019 & Mujer & $\begin{array}{c}\text { Trabajadora } \\
\text { social }\end{array}$ & $\begin{array}{l}\text { Desde el inicio } \\
\quad(2001)\end{array}$ & Dependencia & Individual \\
\hline E4A_2019 & Mujer & $\begin{array}{l}\text { Emprendedora } \\
\text { (promoción de } \\
\text { la mujer) }\end{array}$ & $\begin{array}{l}\text { Desde el inicio } \\
\quad(2006)\end{array}$ & $\begin{array}{l}\text { Actividades } \\
\quad \text { (antes } \\
\text { presidenta) }\end{array}$ & Individual \\
\hline E4B_2019 & Mujer & $\begin{array}{l}\text { Técnica de } \\
\text { laboratorio }\end{array}$ & $\begin{array}{l}\text { Desde el inicio } \\
\text { (2006) }\end{array}$ & Sociosanitaria & Individual \\
\hline E5_2019 & Hombre & $\begin{array}{c}\text { Gestión } \\
\text { Cooperativa } \\
\text { Vivienda }\end{array}$ & $\begin{array}{c}\text { Desde el inicio. } \\
\text { Convivencia } \\
\text { desde } 2018\end{array}$ & Jardinería & Individual \\
\hline E6_2019 & Mujer & Psicóloga & Desde 2014 & Dependencia & Individual \\
\hline E7_2011 & Hombre & $\begin{array}{l}\text { Sociólogo. } \\
\text { Profesor } \\
\text { Facultad de } \\
\text { Economía }\end{array}$ & $\begin{array}{l}\text { Unos años } \\
\text { después del } \\
\text { inicio }\end{array}$ & $\begin{array}{l}\text { Mediación y } \\
\text { resolución de } \\
\text { conflictos }\end{array}$ & Individual \\
\hline E8_2013 & Mujer & Profesora & $\begin{array}{l}\text { Desde el inicio } \\
\text { hasta } 2017\end{array}$ & $\begin{array}{c}\text { Consejo Rector } \\
\text { y C. Puertas } \\
\text { abiertas }\end{array}$ & Individual \\
\hline E9_2013 & Hombre & Ingeniero & $\begin{array}{l}\text { Desde el inicio } \\
\text { hasta } 2017\end{array}$ & Obra & Individual \\
\hline
\end{tabular}

\section{ANÁLISIS DE RESULTADOS}

\section{Contexto de institucionalización de los cuidados y de la dependencia}

La institucionalización de los cuidados y de la dependencia, con la aprobación de la Ley 39/2006, de 14 de diciembre, de Promoción de la Autonomía Personal y Atención a personas en situación de dependencia (LAAD), se planteó como un hito histórico en el ámbito de la protección social y de los servicios sociales. Incluso se llegó a hablar de que podía constituir el cuarto pilar del estado de bienestar ${ }^{3}$, junto con las pensiones, la educación y la sanidad. Sin embargo, su puesta en marcha demuestra ser una solución insuficiente y focalizada que interpela a la responsabilización individual ante las situaciones de dependencia. La LAAD atiende con una cobertura limitada únicamente las situaciones más graves, reforzando la responsabilidad en los hogares y, dentro de ellos, en las mujeres. Además, esta intervención incluye escasas medidas para la promoción de la autonomía personal de las personas con

3 IMSERSO (2004) Libro Blanco de Atención a Personas en Situación de Dependencia. Ministerio de Trabajo y Asuntos Sociales. 
Cuidados comunitarios y gobierno común de la dependencia: las viviendas colaborativas de personas mayores

necesidades de apoyos, dando prioridad a intervenciones médicas y rehabilitadoras sobre las personas en situación de dependencia (Serrano et al., 2013; Artiaga, 2015).

En la formulación de la LAAD, la dependencia se considera un estado permanente e intrínseco a algunas personas por su distancia a una norma de autonomía que establece cuáles son las capacidades deseables y óptimas.

El estado en el que se encuentran las personas que, por razones ligadas a la falta o pérdida de autonomía fisica, psíquica o intelectual, tienen necesidad de asistencia y/o ayudas importantes para realizar las actividades de la vida cotidiana (Recomendación № (98) 9, Ley 39/2006).

Las personas dependientes serían aquellas alejadas de un referente óptimo de autosuficiencia muy dificil de conseguir para gran parte de la población (Pié, Romañach y Ros, 2012). Estos marcos de comprensión que vinculan la dependencia con carencias individuales de algunos tipos de sujetos evocan soluciones sobre los sujetos y no tanto para los sujetos, ocultando así el carácter social de la dependencia. Una de las soluciones que propone la Comisión Europea ${ }^{4}$ ante estas situaciones es la puesta en marcha de acciones correctivas que traten de rehabilitar a las personas dependientes y reducir al máximo, prevenir y evitar estas situaciones.

La rehabilitación debe permitir a la persona dependiente volver a ser tan autónoma como sea posible en el plano psíquico, fisico y social (Recomendación № (98) 9, p. 5).

Se deberán llevar a cabo intervenciones de carácter preventivo que eviten la aparición de situaciones de dependencia, prevengan su agravamiento y reduzcan al máximo su grado de evolución (Recomendación № (98) 9, p. 5).

Estos marcos discursivos que vinculan la dependencia con una anomalía individual a rehabilitar o corregir niegan que la dependencia o la fragilidad de los cuerpos está detrás de lo que significa ser humano y que puede acentuarse por el contexto social. Al ser considerada una cuestión individual y aislable, se oculta el carácter social de estas situaciones, animando a los sujetos a responsabilizarse de sí mismos ante lo que se considera su propia vulnerabilidad. Algunas nociones en auge en los marcos de la regulación de lo social, como la de envejecimiento activo, se ubican dentro de estos marcos individualizadores de comprensión de la dependencia que instan a los sujetos a movilizar todas sus habilidades, en este caso, para luchar contra el paso del tiempo en una etapa de la vida humana como la vejez.

Una respuesta fundamental a este rápido cambio de la estructura de edades consiste en promover la creación de una cultura del envejecimiento activo como un proceso permanente (Decisión № 940/2011/UE, p. 246-5).

En definitiva, entender la dependencia como un rasgo individual de sujetos considerados deficitarios, invisibiliza las tensiones entre la fragilidad de la vida humana y un modelo social que da la espalda a la vulnerabilidad, agudizando aún más las situaciones de dependencia.

Asimismo, en relación con la provisión de cuidados, podemos decir que con la LAAD se reconfiguran las relaciones entre los distintos actores: hogares, mercado, instituciones públicas y comunidad, pero esto no implica una responsabilización colectiva en su cobertura.

Las organizaciones empresariales adquieren un peso relevante en la provisión de cuidados tras el impulso de la LAAD, mediante la gestión directa o la subcontratación pública del Servicio de Ayuda a Domicilio (SAD) o de los servicios residenciales (Comas D'Argemir,

\footnotetext{
4 Recomendación № (98) 9 del Comité de Ministros a los Estados miembros relativa a la dependencia (18 de septiembre de 1998).

Decisión № 940/2011/UE del Parlamento Europeo y del Consejo, de 14 de septiembre de 2011, sobre el año europeo del envejecimiento activo y de la solidaridad intergeneracional (2012).
} 
2015). También tras la puesta en marcha de la prestación monetaria para el pago de servicios profesionales a empresas, así como del criterio del copago o repago, que requiere la participación de las personas usuarias en el pago de un porcentaje del servicio recibido. Con la subcontratación empresarial, se observan espacios híbridos de provisión de los cuidados que favorecen el desplazamiento de las fronteras entre lo profesional y lo familiar o lo público y lo privado. Por ejemplo, con SAD, los cuidados profesionales entran al ámbito privado de los hogares a partir de la subcontratación pública a empresas. Sin embargo, todo esto no supone la desfeminización o la desprivatización de los cuidados, sino más bien un nuevo nicho de negocio para las empresas en el sector de la dependencia y los cuidados.

Además, el alcance de las prestaciones y servicios que se conceden con la LAAD es muy limitado, con la consiguiente derivación de las responsabilidades de cuidados a las mujeres dentro de los hogares. Los retrasos en la concesión de las ayudas, el reducido alcance de estas y su carácter subsidiario, sumado a las políticas austericidas ${ }^{5}$ implementadas tras el estallido financiero del 2008 (Gálvez, 2013), ponen en cuestión la universalidad en el acceso y evidencian su carácter residual. La prioridad que se le otorga en la concesión de las ayudas a la prestación económica para cuidados en el entorno familiar ${ }^{6}$ (Agulló et al., 2011) también contribuye a la refeminización de los cuidados, bien a través de cuidados de mujeres de la familia o de la delegación de los cuidados en otras mujeres, generalmente migrantes (Pérez y López, 2011; Díaz Gorfinkel, 2008).

En este contexto mercantilización y feminización de los cuidados y de modelos de atención a la dependencia deficitarios y rehabilitadores surgen iniciativas desde la comunidad para organizar la cobertura de las necesidades de apoyo durante la vejez de sus participantes. A continuación, observamos su papel en la producción y distribución de bienestar y de cuidados, así como en la resignificación de la cuestión de la dependencia.

\section{El papel de la comunidad en el gobierno de lo común y del bienestar}

La comunidad constituye un objeto de estudio relevante en nuestros días dado su papel fundamental en la provisión del bienestar en contextos de estados deficitarios o con dificultades para que la vida se siga manteniendo en buenas condiciones para toda la población (Gutiérrez, 2017; León, 2017; García Domínguez, Pérez y Artiaga, 2017; Serrano et al., 2019). También es relevante su contribución en la formulación de nuevos imaginarios y prácticas de organización social del bienestar. Amplían la comprensión de lo económico, más allá de los mercados capitalistas y de la acumulación de capital como fin último, lo político, más allá de la intervención del estado y las políticas públicas y, lo familiar, más allá de la familia de sangre o legalmente constituida. Para observar el gobierno de la comunidad en la producción y distribución de bienestar, partimos de la experiencia de las viviendas colaborativas de personas mayores y, en particular, del estudio de caso del centro social de mayores Trabensol.

En primer lugar, en relación con lo político, esta iniciativa se formula como respuesta ante las carencias institucionales que presentan los servicios residenciales que ofrece el Sistema de Atención a la Dependencia y Promoción de la Autonomía (SAAD). Las personas entrevistadas consideran que la iniciativa de Trabensol da respuesta ante el carácter focal de los servicios residenciales, que permiten acceder únicamente a las personas en situaciones

5 Real Decreto Ley 20/2012, de 13 de julio, de medidas para garantizar la estabilidad presupuestaria y de fomento de la competitividad. Establece, entre otros, el límite en las horas máximas que se conceden del servicio de ayuda a domicilio, la incompatibilidad en la solicitud de varios servicios, la reducción de la cobertura mínima garantizada o la suspensión de la cotización a la seguridad social para cuidadoras familiares. Esta última medida ha sido revisada con el Real Decreto-ley 6/2019, de 1 de marzo, de medidas urgentes para garantía de la igualdad

6 Artículo 18, Ley 39/2006. 
Cuidados comunitarios y gobierno común de la dependencia: las viviendas colaborativas de personas mayores

más graves, cuando ya no se puede garantizar su atención ni a través del mercado ni de la familia. Además, comentan que los servicios residenciales suponen una merma en la soberanía política de las personas durante su vejez, desempeñando el papel de guarderías de mayores.

Se trata de ser un testimonio de la falta de recursos públicos para un segmento de la población, los cuales no pueden optar a los servicios públicos existentes por estar destinados a los más desfavorecidos (E_7. Hombre_2011)

Es una guardería. Entonces no... Hacen lo que pueden eh... (E_2. Mujer_2019)

Trabensol surge como respuesta a los déficits institucionales, pero su intención no es distanciarse de las instituciones sino, más bien, interpelar al estado y las administraciones públicas para que reconozcan legalmente la función social de los alojamientos colaborativos en el ámbito de los servicios de atención a la dependencia. Este reconocimiento ${ }^{7}$ se considera que podría reducir los costos económicos para las personas participantes y hacer más universalizable la iniciativa.

y que haya un reconocimiento legal y social del papel que estamos cubriendo (...) Yo creo que es un modelo que debería ser más extendible también si las administraciones facilitaran, por ejemplo, permisos o suelo más barato, o un cierto reconocimiento (E_3. Mujer_2019).

Asimismo, esta iniciativa es un ejemplo de democracia directa donde las decisiones de los asuntos cotidianos de las personas que integran la cooperativa se toman en asamblea y se organizan comisiones de trabajo y actividades para atender a las distintas necesidades que se plantean (de mantenimiento, biblioteca, economía, dependencia, relaciones laborales, comunicación, acogida, excursiones, jardinería y huerto, decoración, fondo solidario, sociosanitaria, etc.). Las personas integrantes valoran la capacidad de apropiarse del gobierno de sus asuntos sin tener que delegar las decisiones sobre sus vidas en otras personas, lo que les permite alcanzar una mayor soberanía política durante su vejez.

en las asambleas multitudinarias recogíamos inquietudes de la gente: “¿Cómo se va a pagar esto?" “Qué va a costar?" “Nos podemos llevar mascotas?" “Qué haremos si nos ponemos malos?" "Y el día que nos muramos, ¿qué pasará con...?” (E_3. Mujer_2019)

Porque la residencia es: tú vas y te ponen en tal sitio y te dicen que a tal hora comer, a tal hora cenas, a tal hora tienes el paseo o te levantas. Es decir, que lo tienes todo estructurado, tú no puedes opinar... (E_9. Hombre_2013)

Se parte de los intereses individuales de las personas cooperativistas para alcanzar comunes compartidos con los que todas las personas puedan convivir, lo que supone un esfuerzo por trabajar los desacuerdos e integrar las distintas sensibilidades e implica cierta cultura política y experiencia en este modo de funcionamiento.

en el compartir tengo que respetar tu mapa, que es diferente al mío, pero que, si intentamos acercarnos, podemos hacer un mapa interesante (E_4B. Mujer_2019)

El bienestar se garantiza a partir de una trama compleja de relaciones de intercambio, negociación y reciprocidad que exigen un aprendizaje y la puesta en marcha de habilidades de sociabilidad. Se recuperan maneras de hacer que implican la puesta en marcha de identidades relacionales frente a la fantasía de la individualidad (Hernando, 2012). Permiten habitar la propia experiencia alejadas del mito de la autosuficiencia y teniendo en cuenta la interdependencia como mecanismo de supervivencia para que la vida continúe (Martín Palomo, 2016; Pérez Orozco, 2014). Se trata de construir una individualidad en relación con el resto y a partir del resto.

hay una ayuda mutua en cuanto dices: "Hola". Enseguida aparecen siete (E_2. Mujer_2019)

\footnotetext{
7 Por ejemplo, señalan que las instituciones públicas podrían contribuir a través del abaratamiento del precio del suelo, otorgando la concesión directa de las ayudas de la LAAD (prestación monetaria para contratación de servicios) o contratando directamente los servicios sociosanitarios que prestan.
} 
porque sí quería un proyecto que hiciera una superación de esta sociedad individual (E_5. Hombre_2019)

Queremos un sitio donde podamos tener una individualidad, y una independencia, y también vivir en común una serie de cosas (E_4A. Mujer_2019)

En segundo lugar, en relación con lo económico, se revisan algunas de las normas morales o legitimidades de la economía de mercado. Por ejemplo, la propiedad privada de la vivienda se sustituye por la propiedad cooperativa, que proporciona el derecho de cesión de uso de cada apartamento y de los espacios comunes a cada cooperativista. Lo consideran fundamental para evitar la especulación inmobiliaria y preservar los objetivos del proyecto, incluso habiendo relevo generacional.

son todos de cesión de uso, eh, todos. Así, desde luego, sí que frenas mucho la especulación urbanística (E_2. Mujer_2019).

También se asumen algunas contradicciones y ambivalencias al situarse en el contexto de un modelo de economía de mercado capitalista como, por ejemplo, la participación en el proyecto aún depende en gran parte de la capacidad de compra en el mercado, tanto en el acceso a la vivienda como en la prestación de servicios.

El carácter colectivo del proyecto implica que se puedan desmercantilizar gran parte de las tareas y servicios y reducir la dependencia monetaria, sin embargo, el acceso a la vivienda aún depende en gran parte de sus ingresos y gran parte de los servicios requieren de contratación externa a empresas privadas (comidas, lavandería, ayuda a domicilio, fisioterapia, terapia ocupacional, entre otras). Se observan tensiones y ambivalencias en un debate que se prolonga durante años sobre la externalización de los servicios y que se ve dificultado por la falta de apoyo institucional.

no podemos contratar directamente porque no tenemos el reconocimiento de empresa gestionadora de servicios, ¿me explico?, ese es uno de los grandes problemas... (E_3. Mujer_2019)

Al ser una contrata son ellos las que pone las condiciones. Nosotros vigilamos, por supuesto nos tienen que presentar que estén dados de alta, etcétera, pero ahí nosotros no nos metemos, de momento hasta que veamos que nos interesa más contratar directamente (E_8. Mujer_2013).

En tercer lugar, en relación con la familia, Trabensol fomenta relaciones de intimidad y compromisos afectivos y solidarios que van más allá de la familia de sangre o legalmente constituida. Se considera que es necesario habitar esta etapa de la vida en colectivo, tanto por ganar soberanía política sobre cómo vivir la vejez, como por los cambios que se han producido en las familias, en los mandatos de género y por las exigencias temporales del mercado laboral.

Yo creo que es mucho más positivo que el otro modo, de que decidan por ti: ¿dónde tienes que ir? ¿a qué edad? ¿Y qué hay que hacer? Y que lo decidan los hijos. Porque los hijos pueden hacerlo con todo el cariño del mundo, pero son sus ideas pero no es la mía (E_9. Hombre_2013).

(...) están en sus trabajos, están en sus niños, están en sus cosas (...) eso fue la generación anterior (E_2. Mujer_2019).

(...) antes eran las labores de las mujeres, sus labores (E_4A. Mujer_2019).

En definitiva, estas iniciativas comunitarias plantean un modo de entender lo político, lo económico y lo familiar que contribuye a desplazar las fronteras de la actual organización social del bienestar y a adquirir nuevos modos de gobierno sobre la propia vida durante vejez. 


\section{Marcos de sentido en torno a la dependencia y la vejez}

La regulación política de la dependencia que se pone en marcha con la LAAD no solo pretende dar respuesta al problema de la dependencia o responder a la función ideal que se establece en la ley (Foucault, 2008), sino que más también contribuye a difundir una manera de entender lo que significa la dependencia y quiénes son los sujetos considerados como tales. En la LAAD se considera dependientes a las personas mayores o con alguna diversidad funcional o cognitiva, cuyas características físicas se utilizan para definir su esencia, esto es, ser personas dependientes. Este uso metonímico del concepto de dependencia se asienta en ejes de poder y jerarquización social que clasifican a los sujetos en función de una norma deseable de autonomía.

Las personas integrantes de Trabensol viven la dependencia y la vejez como una cuestión social que requiere desplazar la mirada más allá del sujeto y ampliar la visión a la vida social donde este se inserta. Los sujetos mayores tienen necesidades específicas, pero estas no pueden disociarse del entramado de relaciones sociales que habitan. Las inadaptadas infraestructuras de las ciudades y de las viviendas, la fragmentación de las relaciones sociales y de la vida vecinal, así como la organización en familias nucleares vulnerabilizan a las personas mayores generando aislamiento, soledad e incrementando la dependencia. Es decir, consideran que, si no se integran las necesidades de esta etapa de la vida en nuestros modelos de organización social, las situaciones de dependencia se agravan. Como señala un participante de Trabensol:

Normalmente los vecinos ni se hablan, ni se conocen. Entonces, ¿tú cómo envejeces ahí? Pues aislao, solo, viendo la televisión ( E_7. Hombre_2011)

En Trabensol, el sostenimiento que ofrece una vida en colectivo y los mecanismos de solidaridad que se despliegan, hacen que disminuya la responsabilidad depositada sobre sus cuerpos y sus experiencias individuales, aumentando su autonomía personal.

Y luego hay otra señora también con un Alzheimer más leve, diríamos. Por ejemplo, puede llevar una vida totalmente autónoma porque vive en Trabensol. Si no viviera en un espacio así, no podría. Porque, por ejemplo, aquí sale por el pueblo, va a pasear, sabe volver. Si se pierde alguien la va a encontrar y la va a traer (...) No se va a encargar de la ropa, no se va a encargar de la comida. Esto le permite tener una vida autónoma (E_1. Mujer_2019).

Este proyecto también permite a sus integrantes politizar esta etapa de la vida y decidir sobre ella, recuperando la soberanía política del sujeto mayor (Mogollón y Fernández, 2019). Se problematiza la idea de que ser mayor signifique ser intervenido y asistido por el resto, ya sea por instituciones públicas, privadas o por la familia.

Pues por buscar un modo de ser mayor diferente, que pretende, por un lado, despegarse de los hijos y poder seguir llevando una vida autónoma (E_7. Hombre_2011)

$Y$ entonces, en el ánimo de los que empezaron en este grupo y el de los que nos fuimos uniendo estaba que no, que esta es una etapa muy importante de nuestra vida y que no nos la gestiona nadie (E_8. Mujer_2013)

Para las personas entrevistadas, decidir sobre esta etapa de la vida supone que el bienestar no solo dependa de intervenciones terapéuticas o sobre el cuerpo sino también de la calidad de las relaciones humanas y de la posibilidad de llevar a cabo un proyecto social, transformador y solidario. Vivir una vejez digna implica vivirla entre iguales, es decir, con personas con las que se ha compartido una generación, con las que pueda existir una cierta comprensión y empatía por estar en el mismo momento vital, con similares necesidades, gustos y deseos.

de cara al futuro me proporciona la tranquilidad de que voy a vivir, además, en un entorno de iguales. Quiero decirte, que puedes tener muy buena relación con los hijos, yo la mantengo, por supuesto, pero es otra generación. No habla tu lenguaje, no ha tenido tus experiencias, no 
sé qué. Entonces aquí sales y sabes de lo que estás hablando. Si hablas de las carreras del 68, de no sé qué, sabes de lo que estás hablando (E_3. Mujer_2019)

la calidad y cualidad de las relaciones humanas sean unos de los factores que te llevan a estar bien (E_7. Hombre_2011)

En la construcción política de ese sujeto mayor también se encuentran algunas dificultades. Las renuncias que supone impulsar este proyecto, la tradición familiarista o la falta de cultura cooperativista pueden ser obstáculos para participar en este tipo de iniciativas (Mogollón y Fernández, 2019). Asimismo, algunas personas entrevistadas señalan que la politización de la vejez no resulta fácil porque requiere una cierta anticipación, previsión y aceptación del envejecimiento, que es una circunstancia que se vive con cierto distanciamiento y desidentificación y, cuando esta etapa avanza, ya es demasiado tarde para poder involucrarse en este cometido. La persona entrevistada relata como algunas personas de Trabensol no quieren entrar al sistema de atención a la dependencia que han creado para que no se les identifique como dependientes por el grupo.

Entonces: "Yo no necesito nada". "Yo no necesito nada". Entonces, ocho personas... Al final quedaron ocho personas que parece ser que sí eran posibles de atender (E_2. Mujer_2019).

Entonces, hay como una resistencia a que me vean con esos (E_3. Mujer_2019).

Se observan ambivalencias entre, por un lado, el reconocimiento de la dependencia durante la vejez como una etapa más de la vida y, por otro lado, la dificultad para su aceptación o incluso su negación. La vejez no es una etapa con límites bien definidos y, además, está cargada de asunciones en torno a las carencias, deficiencias o pérdidas que supone con respecto a la vida adulta. Implica transformaciones en las habilidades fisicas y cognitivas y cambios en las necesidades y modos de vida, que además no están integrados ni reconocidos socialmente.

Hay unas adaptaciones que las haces de forma espontánea y bien (...) Ni a mí se me ocurrirá ponerme la ropa de los 15 años. Eso lo vas aceptando bien, pero hay otras cosas que cuestan mucho más (E_1. Mujer_2019).

Es muy dificil en las personas mayores admitir esta... Las deficiencias, porque claro, ha sido... Toda la gente ha sido muy activa. Aquí hay mucha gente muy pro... Profesionales (...) Entonces, claro, parece que no, pero esto te da un sesgo determinado, ¿no? Y es más dificil el pedir y demás, ¿no? (E_2. Mujer_2019).

Muchas veces, la transición a la vejez es incluso un proceso que resulta imperceptible para la persona que lo habita ya que los cambios pueden ser muy graduales.

un día te mareas, otro día el equilibrio lo empiezas a tener un poquito más chungo, te cuesta andar, te da la impresión de que ya no ves bien para conducir. Son cosas que van muy paulatinas (E_1. Mujer_2019).

Además, el paradigma de la activación, que se inserta en el ámbito de la regulación laboral para promover la adaptabilidad de los sujetos a las exigencias del mercado y penalizar la dependencia (Fernández y Serrano, 2014), es un concepto que se traslada al ámbito de las políticas sociales con la noción de envejecimiento activo. Este significante se considera que integra una mirada edadista y asimilacionista de la vejez (Mogollón y Fernández, 2019) que interpela a las personas mayores a estar activas en el mercado laboral, extendiendo su edad de jubilación, para reducir los costes de lo que se considera el riesgo de longevidad (FMI, 2012).

Este significante también se incorpora al lenguaje de algunas experiencias comunitarias como Trabensol para referirse a la realización de múltiples actividades en la vejez y, en ocasiones, puede convertirse en un mandato que niega la fragilidad de los cuerpos y de la vida.

Pero hay un rechazo a aceptar los límites, a que... A ver, aqui se ha vendido mucho la moto de que hay que envejecer activos, saludables, no sé qué, y entonces, que íbamos a estar todos 
Cuidados comunitarios y gobierno común de la dependencia: las viviendas colaborativas de personas mayores

tocando castañuelas hasta que tocaba morirte, eso sí, la muerte no se negaba del todo, pero bueno, te tenías que morir algún día porque había que morirse. Pero ibas a estar con la juerga hasta el momento pum, de caerte (E_3. Mujer_2019).

Aunque el acento está puesto en la consecución de una vejez digna y el reconocimiento de la dependencia, en ocasiones, se pueden confundir el activismo y la soberanía política con la activación de los sujetos y el mito de la autosuficiencia, produciéndose una cierta jerarquización entre los sujetos considerados válidos y no válidos.

Además, a Trabensol únicamente pueden incorporarse personas entre 50 y 70 años y que no tengan una dependencia grave. Se considera que la incorporación de miembros más mayores o con gran dependencia pondría en riesgo la sostenibilidad económica y humana del proyecto. Por un lado, porque en el contexto capitalista y de falta de apoyo institucional en el que se inserta esta iniciativa, la cobertura de cuidados específicos dependen en gran parte de la capacidad económica de los integrantes del proyecto. Por otro lado, porque la esencia del proyecto depende de la participación y activismo de sus integrantes ya que no quieren ser un proyecto asistencial.

Pero es que esta casa es muy activa (...) Además de ir a Madrid todo el día... En Madrid, en no sé qué, en para allá, para acá, de vacaciones (...) La gente está bien. Entonces claro, eso... Por eso no queríamos... No queremos gente muy mayor... Coger a gente muy mayor (...) De 50 a 70 (E_2. Mujer_2019)

Lo que en principio pensamos fue que la gente que entrase que casi todos fuésemos válidos. No podíamos empezar ya a echar a andar esto con necesidades de personas que tienen que tener ayudas porque eso económicamente íbamos a ver que no era posible. Ahora ya, estando aquí, en la medida en que se van haciendo mayores y vamos necesitando cosas, la cooperativa vamos dotando del personal que se necesite (E_9. Hombre_2013).

Esta propuesta de envejecimiento activista (Mogollón y Fernández, 2019) de Trabensol experimenta dos tensiones. Por un lado, la misión activista y de autoorganización del proyecto requiere cuerpos activos, pudiendo invisibilizar en ocasiones la fragilidad de los cuerpos y exigir un ideal de autosuficiencia condicionado por el paradigma del envejecimiento activo. Por otro lado, este proyecto supone una contribución importante a la politización de la vulnerabilidad de la vida como potencia colectiva y no tanto como debilidad, siendo pionero en la creación de un Sistema de atención a la dependencia propio, como veremos en el siguiente apartado.

\section{La organización comunitaria del cuidado}

Los cuidados adquieren un peso muy relevante en la vivienda colaborativa Trabensol, garantizando cotidianamente el bienestar físico y emocional de las personas integrantes. Se satisfacen las necesidades de cuidados que se han definido colectivamente a través de servicios profesionales (comida, limpieza, fisioterapia, lavandería, pilates, yoga, gimnasia de espalda, podólogo, etc.) o de prestaciones que ofrecen las propias personas de la cooperativa (enfermería y asignación de medicamentos, salidas a la naturaleza, bádminton, taller de costura, cinefórum, etc.). Además, existen mecanismos informales de cuidado y solidaridad colectiva, como se observa a continuación.

$Y$ aquí cuando tienes una gripe, siempre hay una buena vecina que te trae un caldito, o un buen vecino, que también los hay, que te trae el caldito del comedor, que te dice: "Oye, que te traigo de la farmacia lo que necesitas...." Entonces, hay esa solidaridad de los pequeños servicios inmediatos (...) (E_3. Mujer_2019).

El otro día, cuando sabíamos que a -un cooperativista- le iban a operar, ya teníamos programado una cena comunitaria para el sábado. Pero dijimos: "Que mejor que adelantarla y achucharle" (...) Le hicimos unas canciones, le hicieron un versito (E4_AB. Mujer_2019). 
Mi cuñada ha estado sana siempre, pero le entro un cáncer y se ha muerto. Esos meses aqui ha estado como si tuviera 50 hermanos, no voy a decir 80, pero hermanos de verdad. Gente que se ha quedado toda la noche cuando ha estado ahi para que mi hermano descansara (E_5. Hombre_2019).

Los cuidados se entienden en un sentido amplio como las acciones e interacciones que contribuyen a regenerar tanto la vida personal, encarnada en el propio cuerpo, como la vida colectiva, que se expresa en la buena convivencia y el bienestar común. El día a día en esta comunidad implica involucrarse en un entramado de afectos, relaciones y acciones para favorecer el bienestar común más allá del bienestar propio o de las personas más cercanas. Existe una dimensión colectiva del cuidado a partir de la cual la supervivencia de todas las personas del grupo es una prioridad y el bienestar es un concepto relacional.

Desde Trabensol también se ha puesto en marcha un sistema de atención a la dependencia que consiste en ofrecer una cartera de servicios específicos a personas en situación de mayor dependencia y que se financia a través de una mutua solidaria sobre la que trabaja una comisión creada para ello. Se decide impulsar esta iniciativa de cara a garantizar unos cuidados dignos para las personas usuarias con mayores necesidades de apoyo y también unas condiciones dignas de acompañamiento para las personas que son cuidadoras principales y para el resto cooperativistas.

empezamos a tener personas con muchas dificultades, que recaía el cuidado en su pareja, que estaba muy asfixiada-como es normal-; otra que estaba sola (...) ya no podía transferirse ella sola a la cama y empezó a estar muy dependiente de la buena voluntad de los vecinos. Y claro (...), algunos decíamos: "No podemos depender eternamente de la buena voluntad de los vecinos", que pueden estar tan pochos como tú (E_3. Mujer_2019)

El modelo de mutua solidaria ${ }^{8}$ que financia la iniciativa se elabora por las personas que integran Trabensol y su sostenimiento económico depende de la aportación solidaria de todas las personas cooperativistas. Con este proyecto se politiza la transformación de los cuerpos y sus habilidades y la vulnerabilidad se integra como parte de la comprensión de lo político y de lo común en Trabensol.

A ver, el servicio se ha organizado con una idea mutual. De forma que todos los cooperativistas pagamos una parte fija, que es el $70 \%$ del costo de la de la dependencia de todo el mundo. (E_1. Mujer_2019).

Estas iniciativas contribuyen a que la provisión de cuidados en Trabensol traspase las fronteras de lo doméstico, lo familiar y lo privado y se colectivice. No obstante, en este contexto de responsabilización social en los cuidados, se plantea un dilema que tiene que ver con el establecimiento de límites entre el espacio individual y el colectivo para que no se desborden algunas parcelas individuales.

El espacio propio existe físicamente, ya que los apartamentos son individuales o de parejas, pero en las diferentes situaciones es necesario identificar, negociar y establecer dónde están las fronteras para cada quién. El establecimiento de las fronteras del cuidar está en continua revisión, existiendo una diversidad de posiciones en la definición de los compromisos entre las y los cooperativistas. Hasta dónde se comparte la vida, cuánto se puede pedir al resto o cuál es el espacio de lo íntimo y personal, son algunas de las cuestiones que se ponen a dialogar en sus relatos y vivencias. También se reflexiona acerca del cuidado directo del cuerpo, que saca a la luz dimensiones del cuidado que no resultan tan agradables de atender.

8 El modelo de mutua solidaria de Trabensol se decide entre más de diez modelos que se presentan en asamblea. Se establece una cartera de servicios a decisión de las personas usuarias. Dentro de estos servicios hay algunos que son individuales (atención en el domicilio -aseo, vestirse, fregar, etc.-) y otros que son colectivos (sacar residuos, compra, recoger ropa de la lavandería, repartir la medicación, acompañamiento en el vaso terapéutico, terapia ocupacional). Además, se establecen algunos criterios de ratios de personal y número de horas de atención que se otorga a cada persona equivalente a los que se definen en la LAAD para centros residenciales concertados por si en un futuro obtienen el reconocimiento (4.5 profesionales/10 usuarias/os y Grado 1: 1 hora de atención. Grado 2: 3 horas. Grado 3: 5 horas). En función del número de dependientes, el número de horas que le corresponde a cada quién y el coste del servicio profesional se define el gasto total en cada periodo. El $70 \%$ del gasto total se reparte entre las personas de la cooperativa que pagan una cuota general y, las personas usuarias, además pagarán una cuota específica resultado del reparto del $30 \%$ del coste total ponderado en función del grado de dependencia y, por tanto, del número de horas de servicio que van a requerir. 
Cuidados comunitarios y gobierno común de la dependencia: las viviendas colaborativas de personas mayores

Porque una cosa es que tú vivas aqui y te pongas enfermo y hoy te lleve yo al hospital y mañana te lleve otro y si hay que traerte un caldito te traiga un caldito, y otra cosa es que cada día te tenga que lavar el culo. Porque ni tú te vas a sentir cómodo ni yo me voy a sentir cómodo (E_6. Mujer_2019).

Lo colectivo en muchas ocasiones desplaza a la externalización del cuidado ya que muchas de las actividades y servicios se ponen en marcha a partir del trabajo cooperativo. Sin embargo, gran parte de las actividades que son demandantes en tiempo, son menos agradables o que exigen ciertas habilidades, se profesionalizan y mercantilizan a partir de la contratación de empresas privadas. Esto abre un diálogo en la cooperativa de hasta qué punto el bienestar digno de las personas cooperativistas se da en función de la provisión de cuidados por parte de empresas que no está claro que garanticen condiciones laborales dignas a sus profesionales. En este caso consideran que la contratación directa podría ser una solución.

una polémica bastante grande porque hay gente que piensa que es mejor que los servicios que nos prestan pues los trabajadores se han contratado directamente. Cuando contratamos a una empresa pues al final nosotros queremos un precio bueno, pero qué ocurre, que ellos aprietan mucho a los trabajadores para llevarse su plusvalía (E_5. Hombre_2019).

En relación con el género del cuidar podemos decir que, especialmente si hablamos de la atención al cuerpo, a la salud física o a las situaciones de dependencia, los cuidados se realizan mayoritariamente por parte de mujeres de la cooperativa, así que podríamos hablar no tanto de una transformación de los roles de género, sino más bien de una distribución más colectiva del cuidado entre mujeres (Mogollón y Fernández, 2019).

Hombre, los hombres cuidan menos, pero sí, sí, pero también, eh. Aquí tenemos, por ejemplo, uno, que está su hermano y está pendiente de su hermano. O sea, que sí. No hay muchos cuidadores hombres, no. Normalmente son mujeres las que cuidan... (E_2. Mujer_2019)

El género de los cuidados se observa en la distribución en tipos de comisiones y grupos de trabajo (por ejemplo, la de dependencia la ocupan únicamente mujeres). Asimismo, las tareas de cuidado indirecto que permiten que el cuidado se haga efectivo, se reparten más equitativamente entre hombres y mujeres. Sin embargo, el cuidado directo sobre los cuerpos, que implica la interacción entre las personas, o la gestión mental del cuidado se realiza mayoritariamente por mujeres, aunque su responsabilidad en cierto modo se minimiza por los mecanismos colectivos implementados.

Por tanto, observamos un modelo híbrido de cobertura de cuidados desde la comunidad que se ubica en una trama compleja de relaciones de alianza y conflicto entre los distintos actores sociales (mercado, familia, estado, comunidad) y los distintos sujetos (hombres y mujeres), que ofrece algunas pistas de cara a elaborar escenarios sociales basados en la interdependencia.

\section{CONCLUSIONES}

En el actual modelo social, la dependencia se considera una desviación de la norma mítica de autonomía y una anomalía a prevenir en un contexto que privilegia a unos pocos cuerpos y vidas, delegando y ocultando los cuidados en la esfera privada y feminizada de los hogares.

La iniciativa comunitaria de vivienda colaborativa de personas mayores, Trabensol, es un laboratorio de ideas y de prácticas que establece otros marcos de lo posible en torno a la dependencia y los cuidados y otras formas de vivir la vejez de manera consciente y negociada, en colectivo. Amplía la comprensión de lo político, lo económico y lo familiar, ofreciendo alternativas más allá de los centros residenciales o del cuidado feminizado y precarizado en los hogares, que reviertan en un bienestar en común o en relación con el resto. Plantea una reorganización del bienestar interpelando a las instituciones públicas y desplazando 
las fronteras entre lo público y lo privado o lo común y lo individual. Del mismo modo, esta iniciativa se inserta en un contexto de mercantilización y feminización de los cuidados del que a veces no puede huir y es coetánea al auge del paradigma de envejecimiento activo, que promueve una ingeniería sobre los cuerpos de los sujetos para que sean activos y productivos hasta el último aliento, lo que a veces se traslada al imaginario de la cooperativa.

El análisis de la iniciativa comunitaria de Trabensol nos permite entender la comunidad no tanto como un horizonte utópico o un paraíso lejano al que nunca podemos acceder pero que anhelamos alcanzar (Garcés, 2013), sino más bien como una realidad presente, compleja e híbrida sobre la que se puede transitar y actuar. Es una experiencia social que plantea nuevos imaginarios y escenarios posibles para la problematización sobre lo que significa hacerse mayor, los límites de los compromisos entre las personas o la negociación de las fronteras de la intimidad, donde poder revisar los mandatos de género de los cuidados o el estigma de la dependencia. Y que, en definitiva, permiten esbozar nuevos horizontes que contribuyan a garantizar una soberanía política sobre nuestros cuerpos y nuestras vidas, en relación con la vida del resto.

\section{REFERENCIAS}

Agulló, C., Arroyo, J., Ema, J. E., Gámez, C., Gómez, E., Jiménez, P., Rodríguez, E., Salvador, M., Orfila, M. y Colina, M. P. (eds.) (2011). Cojos y precarias haciendo vidas que importan. Madrid: Traficantes de Sueños.

Artiaga Leiras, A. (2015). Producción Política de los cuidados y de la dependencia: Políticas públicas y experiencias de organización social de los cuidados (Tesis Doctoral). Universidad Complutense de Madrid.

Bustelo, M. y Lombardo, E. (eds.) (2007). Las políticas de igualdad en España y Europa. Madrid: Editorial Cátedra.

Carrasco, C., Borderías, C. y Torns, T. (eds.) (2011). El trabajo de cuidados. Historia, teoría y políticas. Barcelona: La Catarata.

Comas D Argemir, D. (2015). Los cuidados de larga duración y el cuarto pilar del sistema de bienestar. Revista de Antropología Social, 24, 375-404.

Daly, M. y Lewis, J. (2011). El concepto de social care y el análisis de los estados de bienestar contemporáneos. En C. Carrasco, C. Borderías y T. Torns (eds.), El trabajo de cuidados. Historia, teoría y políticas (pp. 225-252). Madrid: La catarata.

Díaz Gorfinkiel, M. (2008). El mercado de trabajo de los cuidados y la creación de las cadenas globales de cuidado: ¿cómo concilian las cuidadoras? Cuadernos de Relaciones Laborales, 26(2), 71-89.

Durán, M.A. (2018). La riqueza invisible del cuidado. Valencia: Universitat de València.

Esping-Andersen, G. (1999). Social Foundations of Postindustrial Economies. Oxford: University Press.

Fernández, C. y Serrano, A. (coords.) (2014). El paradigma de la flexiseguridad en las políticas de empleo españolas: un análisis cualitativo. Madrid: Centro de Investigaciones Sociológicas (CIS).

Fondo Monetario Internacional -FMI. (2012, abril). Informe sobre la estabilidad financiera mundial (GFSR).

Foucault, M. (2008). Seguridad, territorio y población. Madrid: Ediciones Akal. 
Gálvez, L. (2013). Una lectura feminista del austericidio. Revista de Economía Crítica, 15, 80-110.

Garcés, M. (2013). Un mundo común. Barcelona: Edicions Bellaterra.

García Domínguez, M., Pérez Orozco, A. y Artiaga Leiras, A. (2017). Comunidad, tercer sector y gestión de lo común. Actualización de la serie de documentos ¿Por qué nos preocupamos por los cuidados? (pp: 18-30). Santo Domingo: Centro de Capacitación de ONU Mujeres.

Gutiérrez, R. (2017). Horizontes comunitario-populares. Producción de lo común más allá de las políticas estado-céntricas. Madrid: Traficantes de Sueños.

Hernando, A. (2012). La fantasía de la individualidad. Sobre la construcción sociohistórica del sujeto moderno. Madrid: Katz Editores.

León, C. (2017). Trincheras Permanentes. Intersecciones entre políticas y cuidados. Logroño: Pepitas Ediciones.

López, S. y Platero R.L. (eds.) (2019). Cuerpos marcados. Vidas que cuentan y políticas públicas. Barcelona: Ediciones Bellaterra.

Martín Criado, E. (1998). Los decires y los haceres. Revista de Sociología Papers, 56, 57-71.

Martín Palomo, M.T. (2016). Cuidado, vulnerabilidad e interdependencias. Nuevos retos políticos. Madrid: Centro de Estudios Políticos y Constitucionales.

Mogollón, I. y Fernández, A. (2019). Arquitecturas del cuidado. Hacia un envejecimiento activista. Barcelona: Icaria editorial.

Pérez Orozco, A. (2014). Subversión feminista de la economía. Apuntes sobre el conflicto capital-vida. Madrid: Traficantes de Sueños.

Pérez, A. y López, S. (2011). Desigualdades a flor de piel: Cadenas globales de cuidados. Concreciones en el empleo del hogar y políticas públicas. Madrid: Ed. ONU Mujeres.

Serrano, A., Artiaga, A. y Dávila de León, C. (2013). Crisis de los cuidados, ley de dependencia y confusión semántica. Revista Internacional de Sociología, 61(3), 669- 694.

Serrano, A., Martín, M.Py de Castro Pericacho, C. (2019). Sociologizando la Resiliencia. El papel de la participación socio-comunitaria y política en las estrategias de afrontamiento de la crisis. Revista Española de Sociología, 28(2), 227-247.

Vega, C., Martínez, R. y Paredes, M. (2018). Cuidado, comunidad y común. Extracciones, apropiaciones y sostenimiento de la vida. Madrid: Traficantes de Sueños.

Yin, Robert K. (1984). Case Study Research. Design and Methods. Beberly Hills, CA: Sage Publications. 